\title{
Temperature Dependence of Compression of Natural Rubber-Sulfur Vulcanizates of High Sulfur Content ${ }^{1}$
}

\author{
Charles E. Weir
}

\begin{abstract}
Measurements are reported of the compression of rubber-sulfur vulcanizates in the temperature range $10^{\circ}$ to $81.5^{\circ} \mathrm{C}$ between pressures 1,000 and 10,000 atmospheres. Sulfur contents varied from 10 to 28 percent. Empirical isothermal compression equations are derived. Empirical isobaric equations are derived for specific volume. From the isobars empirical equations of state (pressure-volume-temperature) are derived.
\end{abstract}

\section{Introduction}

In the course of a continuing investigation of the behavior of high-polymeric materials under high pressures, measurements have been made on the pressure-volume-temperature (PVT) relationships of natural rubber-sulfur vulcanizates. This report is concerned with the experimental data obtained on these vulcanizates between $10^{\circ}$ and $81^{\circ} \mathrm{C}$ and between 1,000 and 10,000 atm. Empirical equations have been derived to fit the data.

These data will be used later to obtain a clear answer to the question of the effect of pressure on the second-order or glass transition in polymers and also to evaluate thermodynamic quantities that may prove of interest in correlating molecular or intrachain forces in such polymers. The discussion and calculations involved in these two objectives are deferred for subsequent reports, inasmuch as the compilations of the compression data and the derived equations are quite lengthy. However, the data given here are sufficient for all projected treatment of these objectives.

The behavior of rubber under pressure has been studied by Adams and Gibson [1], ${ }^{2}$ Bridgman [2], Scott [3], and the present author [4]. All previous work except that of Seott [3] was carried out at room temperature and high pressure. The studies of Adams and Gibson, and of Bridgman were conducted on available rubbers of uncontrolled composition, whereas those of reference 4 were carried out on raw, synthetic, and oil-resistant rubbers. Scott [3] worked only in the low-pressure range (maximum 800 bars) but systematically varied composition (sulfur content) and temperature over a wide range. These data of Scott have received a great deal of consideration by current workers in the high-polymer field because they are the only available PVT data on rubbers of various sulfur contents. From consideration of the data presented graphically and in empirical equations by Scott, it would appear that certain questionable conclusions concerning the effect of pressure on the glass transition of rubbers have been drawn by later workers from these data. It must be recalled that the significance of the discontinuities in slopes observed by Scott in the isobars was not

1 This paper is a preliminary summary of work on a project sponsored by the Office of Naval Research project NR 330-032.

${ }_{2}^{2}$ Figures in brackets indicate the literature references at the end of this paper. known at the time the measurements were made and the numerical experimental data were not reported. The present report is designed to supplement the previous studies of Scott.

\section{Preparation and Treatment of Specimens}

The rubber specimens used were prepared from smoked sheet and sulfur and contained no filler. Sulfur was incorporated in the milling operation and the resulting mixture molded into cylinders approximately $\frac{1}{2}$ in. in diameter and 4 in. in length Vulcanization was carried out at $140^{\circ} \mathrm{C}$ for $17 \mathrm{hr}$ to insure combination of the sulfur with the rubber. Sulfur contents reported refer to amounts added because chemical analyses have not been performed. However, as the densities of these specimens agree well with the densities of specimens of corresponding sulfur content reported by Scott, it is considered that practically all the sulfur combined with the rubber. Specimens were prepared to contain sulfur contents between 10 and 28 percent, the use of rubbers of lower sulfur contents being considered impractical in these experiments because of the swelling action of the confining liquid on the rubber.

Prior to each experiment at $21^{\circ} \mathrm{C}$, the weight of each specimen was determined in air, and immediately following compression it was redetermined in the confining liquid - a light petroleum distillate - and in air. The former two weights permitted calculation of the volume and density of the specimen, whereas the latter weight permitted estimation of the quantity of liquid absorbed in the experiment. Densities were determined only at $21^{\circ} \mathrm{C}$, but initial and final weighings in air at $21^{\circ} \mathrm{C}$ were carried out when specimens were run at elevated and reduced temperatures. The quantities of confining liquid absorbed increased with increasing temperature but in no instance reached a magnitude sufficient to cause doubt as to the validity of the experimental data.

\section{Experimental Method}

The apparatus and experimental details have been described in detail previously [5]. An experiment consists in forcing a leak-proof piston into the bore of a heavy-walled cylinder that contains the specimen 
and a manganin pressure gage immersed in a light petroleum distillate. Measurements of the depth of penetration of the piston are made at every 1,000 atm. Combination of these measurements with similar data obtained in an experiment in which the specimen is replaced by a steel bar of comparable volume permits calculation of the compression, $-\Delta V / V_{0}$, of the specimen in terms of that of steel, which is known [6]. This compression is reckoned from the arbitrary reference pressure of 2,000 atm but is calculated in terms of the initial (1 atm) volume of the specimen, $V_{0}$.

At elevated or reduced temperatures the entire hydraulic press and pressure vessel were enclosed in a thermostatted container so designed as to minimize the possibility of setting up thermal gradients in the pressure vessel. Because air was used as the heatexchange medium and the heat capacity of the press and pressure equipment was enormous, at least $48 \mathrm{hr}$ was required for attainment of temperature equilibrium after every change of temperature. Temperatures were measured with a calibrated mercury thermometer set in a well in a massive brass block fastened to and in intimate thermal contact with the pressure vessel. This block was also used to contain the temperature compensating manganin coil [5]. During the course of the measurements a maximum temperature variation of $\pm 0.35 \mathrm{deg} C$ was observed on this thermometer. The enormous heat capacity of the pressure vessel, however, should reduce the temperature variation of the specimen considerably below this figure.

\section{Results of Compression Measurements}

The results of compression measurements of rubber-sulfur vulcanizates at the various temperatures are given in tables 1 to 6 , inclusive. The

TABLE 1. Compression of natural rubber-sulfur vulcanizates at $10.0^{\circ} \mathrm{C}$

$-\Delta V / V_{0}$

\begin{tabular}{|c|c|c|c|c|c|}
\hline \multirow[t]{2}{*}{$\begin{array}{l}\text { Pres- } \\
\text { sure }\end{array}$} & \multirow{2}{*}{$\begin{array}{c}10 \% \mathrm{~s} \\
V_{0}=18.861 \mathrm{~cm}^{3} \\
V^{\prime}=17.918 \mathrm{~cm}^{3}\end{array}$} & \multirow[t]{2}{*}{$\begin{array}{c}13 \% \mathrm{~s} \\
V_{0}=19.117 \mathrm{~cm}^{3} \\
V^{\prime}=18.352 \mathrm{~cm}^{3}\end{array}$} & \multirow{2}{*}{$\begin{array}{c}16 \% \mathrm{~S} \\
V_{0}=17.976 \mathrm{~cm}^{3} \\
V^{\prime}=17.275 \mathrm{~cm}^{3}\end{array}$} & \multicolumn{2}{|c|}{$\begin{array}{c}20 \% \mathrm{~s} \\
V_{0}=18.152 \mathrm{~cm}^{3} \\
V^{\prime}=17.482 \mathrm{~cm}^{3}\end{array}$} \\
\hline & & & & Run 1 & Run 2 \\
\hline $\begin{array}{r}\text { atm } \\
10,000 \\
9,000 \\
8,000 \\
7,000 \\
6,000\end{array}$ & $\begin{array}{r}0.0843 \\
.0758 \\
.0688 \\
.0616 \\
.0531\end{array}$ & $\begin{array}{r}0.0818 \\
.0742 \\
.0668 \\
.0597 \\
.0512\end{array}$ & $\begin{array}{r}0.0834 \\
.0760 \\
.0684 \\
.0614 \\
.0523\end{array}$ & $\begin{array}{r}0.0839 \\
.0759 \\
.0683 \\
.0618 \\
.0530\end{array}$ & $\begin{array}{r}0.0843 \\
.0763 \\
.0689 \\
.0617 \\
.0532\end{array}$ \\
\hline $\begin{array}{l}5,000 \\
4,000 \\
3,000 \\
2,000 \\
1,000\end{array}$ & $\begin{array}{r}.0427 \\
.0315 \\
.0182 \\
.0000 \\
-.0250\end{array}$ & $\begin{array}{r}.0407 \\
.0291 \\
.0160 \\
.0000 \\
-.0214\end{array}$ & $\begin{array}{r}.0414 \\
.0295 \\
.0163 \\
.0000 \\
-.0190\end{array}$ & $\begin{array}{r}.0420 \\
.0297 \\
.0157 \\
.0000 \\
-.0184\end{array}$ & $\begin{array}{r}.0421 \\
.0297 \\
.0158 \\
.0000 \\
-.0186\end{array}$ \\
\hline
\end{tabular}

TABLE 2. Compression of natural rubber-sulfur vulcanizates at $21.0^{\circ} \mathrm{C}$ $-\Delta V / V_{0}$

\begin{tabular}{|c|c|c|c|c|c|}
\hline \multirow{5}{*}{ Pressure } & \multirow{5}{*}{$\begin{array}{c}10 \% \mathrm{~s} \\
V_{0}=19.000 \mathrm{~cm}^{3} \\
V^{\prime}=18.031 \mathrm{~cm}^{3} \\
\rho=0.9947 \mathrm{~g} / \mathrm{cm}^{3}\end{array}$} & \multirow{5}{*}{$\begin{array}{c}13 \% \mathrm{~s} \\
V_{0}=19.262 \mathrm{~cm}^{3} \\
V^{\prime}=18.453 \mathrm{~cm}^{3} \\
\rho=1.0221 \mathrm{~g} / \mathrm{cm}^{3}\end{array}$} & \multicolumn{2}{|c|}{$16 \% \mathrm{~S}$} & \multirow{5}{*}{$\begin{array}{c}18 \% \mathrm{~S} \\
V_{0}=18.586 \mathrm{~cm}^{3} \\
V^{\prime}=17.906 \mathrm{~cm}^{3} \\
\rho=1.0707 \mathrm{~g} / \mathrm{cm}^{3}\end{array}$} \\
\hline & & & Run 1 & Run 2 & \\
\hline & & & $V_{0}=18.141 \mathrm{~cm}^{3}$ & $V_{0}=18.141 \mathrm{~cm}^{3}$ & \\
\hline & & & $V^{\prime}=17.415 \mathrm{~cm}^{3}$ & $V^{\prime}=17.434 \mathrm{~cm}^{3}$ & \\
\hline & & & $\rho=1.0518 \mathrm{~g} / \mathrm{cm}^{3}$ & $\rho=1.0518 \mathrm{~g} / \mathrm{cm}^{3}$ & \\
\hline atm & & & & & \\
\hline $\begin{array}{r}10,000 \\
9,000\end{array}$ & 0.0881 & 0.0864 & 0.0864 & 0.0853 & 0.0868 \\
\hline 8,000 & .0730 & $\begin{array}{l}.0790 \\
.0710\end{array}$ & $\begin{array}{l}.0789 \\
.0705\end{array}$ & $\begin{array}{l}.0776 \\
.0702\end{array}$ & $\begin{array}{l}.0781 \\
.0715\end{array}$ \\
\hline $\begin{array}{l}7,000 \\
6,000\end{array}$ & $\begin{array}{l}.0645 \\
.0555\end{array}$ & $\begin{array}{l}.0624 \\
.0531\end{array}$ & $\begin{array}{l}.0616 \\
.0514\end{array}$ & $\begin{array}{l}.0608 \\
.0515\end{array}$ & $\begin{array}{l}.0624 \\
.0525\end{array}$ \\
\hline \multirow{10}{*}{$\begin{array}{l}5,000 \\
4,000 \\
3,000 \\
2,000 \\
1,000\end{array}$} & .0452 & .0425 & .0408 & .0404 & .0416 \\
\hline & .0337 & .0311 & .0291 & .0286 & .0292 \\
\hline & .0193 & .0173 & .0156 & .0152 & .0160 \\
\hline & $\begin{array}{r}.0000 \\
-.0255\end{array}$ & $\begin{array}{r}.0000 \\
-.0248\end{array}$ & $\begin{array}{r}.0000 \\
-.0201\end{array}$ & $\begin{array}{r}.0000 \\
-.0194\end{array}$ & $\begin{array}{r}.0000 \\
-.0186\end{array}$ \\
\hline & \multicolumn{2}{|c|}{$20 \% \mathrm{~S}$} & \multirow{6}{*}{$\begin{array}{c}22 \% \mathrm{~S} \\
V_{0}=18.541 \mathrm{~cm}^{3} \\
V^{\prime}=17.874 \mathrm{~cm}^{3} \\
\rho=1.0996 \mathrm{~g} / \mathrm{cm}^{3}\end{array}$} & \multirow{6}{*}{$\begin{array}{c}25 \% \mathrm{~S} \\
V_{0}=18.169 \mathrm{~cm}^{3} \\
V^{\prime}=17.515 \mathrm{~cm}^{3} \\
\rho=1.1214 \mathrm{~g} / \mathrm{cm}^{3}\end{array}$} & \multirow{6}{*}{$\begin{array}{c}28 \% \mathrm{~S} \\
V_{0}=18.633 \mathrm{~cm}^{3} \\
V^{\prime}=17.981 \mathrm{~cm}^{3} \\
\rho=1.1422 \mathrm{~g} / \mathrm{cm}^{3}\end{array}$} \\
\hline & Run 1 & Run 2 & & & \\
\hline & & & & & \\
\hline & $V_{0}=18.217 \mathrm{~cm}^{3}$ & $V_{0}=18.217 \mathrm{~cm}^{3}$ & & & \\
\hline & $V^{\prime}=17.543 \mathrm{~cm}^{3}$ & $V^{\prime}=17.561 \mathrm{~cm}^{3}$ & & & \\
\hline & $\rho=1.0877 \mathrm{~g} / \mathrm{cm}^{3}$ & $\rho=1.0877 \mathrm{~g} / \mathrm{cm}^{3}$ & & & \\
\hline 10,000 & 0.0878 & 0.0871 & 0.0876 & 0.0892 & 0.0891 \\
\hline 9,000 & .0801 & .0794 & .0801 & .0803 & .0816 \\
\hline 8,000 & .0722 & .0718 & .0722 & .0737 & .0734 \\
\hline 6,000 & $\begin{array}{l}.0631 \\
.0530\end{array}$ & $\begin{array}{l}.0625 \\
.0528\end{array}$ & $\begin{array}{l}.0628 \\
.0532\end{array}$ & $\begin{array}{l}.0647 \\
.0547\end{array}$ & $\begin{array}{l}.0646 \\
.0545\end{array}$ \\
\hline 5,000 & .0417 & .0415 & .0419 & .0435 & .0434 \\
\hline 4,000 & .0295 & .0291 & .0298 & .0310 & .0312 \\
\hline 3,000 & .0158 & .0155 & .0156 & .0167 & .0170 \\
\hline 2,000 & .0000 & .0000 & .0000 & .0000 & .0000 \\
\hline 1,000 & -.0184 & -.0182 & -.0184 & -.0193 & -.0177 \\
\hline
\end{tabular}


TABLE 3. Compression of natural rubber-sulfur vulcanizates at $38.5^{\circ} \mathrm{C}$

$-\Delta V / V_{0}$

\begin{tabular}{|c|c|c|c|c|}
\hline Pressure & $\begin{array}{c}10 \% \mathrm{~s} \\
V_{0}=19.238 \mathrm{~cm}^{3} \\
V^{\prime}=18.161 \mathrm{~cm}^{3}\end{array}$ & $\begin{array}{c}13 \% \mathrm{~s} \\
V_{0}=19.432 \mathrm{~cm}^{3} \\
V^{\prime}=18.383 \mathrm{~cm}^{3}\end{array}$ & $\begin{array}{c}16 \% \mathrm{~S} \\
V_{0}=18.396 \mathrm{~cm}^{3} \\
V^{\prime}=17.642 \mathrm{~cm}^{3}\end{array}$ & $\begin{array}{c}18 \% \mathrm{~S} \\
V_{0}=18.706 \mathrm{~cm}^{3} \\
V^{\prime}=17.920 \mathrm{~cm}^{3}\end{array}$ \\
\hline $\begin{array}{r}a t m \\
10,000 \\
9,000 \\
8,000 \\
7,000 \\
6,000 \\
\\
5,000 \\
4,000 \\
3,000 \\
2,000 \\
1,000\end{array}$ & $\begin{array}{r}0.0943 \\
.0871 \\
.0795 \\
.0708 \\
.0617 \\
\\
.0510 \\
.0379 \\
.0211 \\
.0000 \\
-.0274\end{array}$ & $\begin{array}{r}0.0896 \\
.0823 \\
.0746 \\
.0658 \\
.0566 \\
\\
.0462 \\
.0341 \\
.0196 \\
.0000 \\
-.0265\end{array}$ & $\begin{array}{r}0.0886 \\
.0809 \\
.0728 \\
.0633 \\
.0536 \\
\\
.0427 \\
.0307 \\
.0169 \\
.0000 \\
-.0240\end{array}$ & $\begin{array}{r}0.0888 \\
.0810 \\
.0727 \\
.0636 \\
.0537 \\
\\
.0425 \\
.0303 \\
.0164 \\
.0000 \\
-.0214\end{array}$ \\
\hline & $\begin{array}{c}20 \% \mathrm{~s} \\
V_{0}=18.309 \mathrm{~cm}^{3} \\
V^{\prime}=17.540 \mathrm{~cm}^{3}\end{array}$ & $\begin{array}{c}22 \% \mathrm{~s} \\
\mathrm{~V}_{0}=18.612 \mathrm{~cm}^{3} \\
V^{\prime}=17.868 \mathrm{~cm}^{3}\end{array}$ & $\begin{array}{c}25 \% \mathrm{~s} \\
V_{0}=18.216 \mathrm{~cm}^{3} \\
V^{\prime}=17.487 \mathrm{~cm}^{3}\end{array}$ & $\begin{array}{c}28 \% \mathrm{~s} \\
V_{0}=18.686 \mathrm{~cm}^{3} \\
V^{\prime}=17.939 \mathrm{~cm}^{3}\end{array}$ \\
\hline $\begin{array}{r}10,000 \\
9,000 \\
8,000 \\
7,000 \\
6,000\end{array}$ & $\begin{array}{r}0.0895 \\
.0819 \\
.0734 \\
.0643 \\
.0543\end{array}$ & $\begin{array}{r}0.0898 \\
.0818 \\
.0739 \\
.0652 \\
.0550\end{array}$ & $\begin{array}{r}0.0908 \\
.0831 \\
.0747 \\
.0658 \\
.0558\end{array}$ & $\begin{array}{r}0.0905 \\
.0830 \\
.0748 \\
.0658 \\
.0558\end{array}$ \\
\hline $\begin{array}{l}5,000 \\
4,000 \\
3,000 \\
2,000 \\
1,000\end{array}$ & $\begin{array}{r}.0431 \\
.0304 \\
.0162 \\
.0000 \\
-.0205\end{array}$ & $\begin{array}{r}.0437 \\
.0309 \\
.0165 \\
.0000 \\
-.0200\end{array}$ & $\begin{array}{r}.0441 \\
.0317 \\
.0165 \\
.0000 \\
-.0199\end{array}$ & $\begin{array}{r}.0445 \\
.0317 \\
.0169 \\
.0000 \\
-.0197\end{array}$ \\
\hline
\end{tabular}

TABLE 4. Compression of natural rubber-sulfur vulcanizates at $50.2^{\circ} \mathrm{C}$

$-\Delta V / V_{0}$

\begin{tabular}{|c|c|c|c|c|}
\hline Pressure & $\begin{array}{c}10 \% \mathrm{~s} \\
V_{0}=19.370 \mathrm{~cm}^{3} \\
V^{\prime}=18.247 \mathrm{~cm}^{3}\end{array}$ & $\begin{array}{c}13 \% \mathrm{~s} \\
V_{0}=19.609 \mathrm{~cm}^{3} \\
V^{\prime}=18.531 \mathrm{~cm}^{3}\end{array}$ & $\begin{array}{c}16 \% \mathrm{~S} \\
V_{0}=18.639 \mathrm{~cm}^{3} \\
V^{\prime}=17.689 \mathrm{~cm}^{3}\end{array}$ & $\begin{array}{c}18 \% \mathrm{~S} \\
V_{0}=18.786 \mathrm{~cm}^{3} \\
V^{\prime}=17.847 \mathrm{~cm}^{3}\end{array}$ \\
\hline $\begin{array}{r}\text { atm } \\
10,000 \\
9,000 \\
8,000 \\
7,000 \\
6,000 \\
\\
5,000 \\
4,000 \\
3,000 \\
2,000 \\
1,000\end{array}$ & $\begin{array}{r}0.0992 \\
.0912 \\
.0836 \\
.0747 \\
.0650 \\
\\
.0533 \\
.0387 \\
.0212 \\
.0000 \\
-.0287\end{array}$ & $\begin{array}{r}0.0932 \\
.0861 \\
.0780 \\
.0696 \\
.0615 \\
\\
.0496 \\
.0368 \\
.0204 \\
.0000 \\
-.0276\end{array}$ & $\begin{array}{r}0.0886 \\
.0812 \\
.0733 \\
.0643 \\
.0547 \\
\\
.0442 \\
.0321 \\
.0181 \\
.0000 \\
-.0264\end{array}$ & $\begin{array}{r}0.0879 \\
.0802 \\
.0723 \\
.0631 \\
.0533 \\
\\
.0426 \\
.0305 \\
.0167 \\
.0000 \\
-.0233\end{array}$ \\
\hline & $\begin{array}{c}20 \% \mathrm{~s} \\
V_{0}=18.448 \mathrm{~cm}^{3} \\
V^{\prime}=17.673 \mathrm{~cm}^{3}\end{array}$ & $\begin{array}{c}22 \% \mathrm{~s} \\
V_{0}=18.654 \mathrm{~cm}^{3} \\
V^{\prime}=17.889 \mathrm{~cm}^{3}\end{array}$ & $\begin{array}{c}25 \% \mathrm{~s} \\
V_{0}=18.297 \mathrm{~cm}^{3} \\
V^{\prime}=17.547 \mathrm{~cm}^{3}\end{array}$ & $\begin{array}{c}28 \% \mathrm{~S} \\
V_{0}=18.772 \mathrm{~cm}^{3} \\
V^{\prime}=18.021 \mathrm{~cm}^{3}\end{array}$ \\
\hline $\begin{array}{r}10,000 \\
9,000 \\
8,000 \\
7,000 \\
6,000\end{array}$ & $\begin{array}{r}0.0898 \\
.0820 \\
.0734 \\
.0648 \\
.0546\end{array}$ & $\begin{array}{l}0.0899 \\
.0827 \\
.0742 \\
.0648 \\
.0548\end{array}$ & $\begin{array}{r}0.0910 \\
.0835 \\
.0753 \\
.0658 \\
.0554\end{array}$ & $\begin{array}{r}0.0910 \\
.0838 \\
.0753 \\
.0661 \\
.0562\end{array}$ \\
\hline $\begin{array}{l}5,000 \\
4,000 \\
3,000 \\
2,000 \\
1,000\end{array}$ & $\begin{array}{r}.0433 \\
.0307 \\
.0165 \\
.0000 \\
-.0213\end{array}$ & $\begin{array}{r}.0436 \\
.0309 \\
.0164 \\
.0000 \\
-.0205\end{array}$ & $\begin{array}{r}.0443 \\
.0312 \\
.0167 \\
.0000 \\
-.0205\end{array}$ & $\begin{array}{r}.0445 \\
.0315 \\
.0169 \\
.0000 \\
-.0210\end{array}$ \\
\hline
\end{tabular}

TABLE 5. Compression of natural rubber-sulfur vulcanizates at $64.0^{\circ} \mathrm{C}$

$-\Delta V / V_{0}$

\begin{tabular}{|c|c|c|c|c|}
\hline Pressure & $\begin{array}{c}10 \% \mathrm{~s} \\
V_{0}=19.522 \mathrm{~cm}^{3} \\
V^{\prime}=18.370 \mathrm{~cm}^{3}\end{array}$ & $\begin{array}{c}13 \% \mathrm{~s} \\
V_{0}=19.668 \mathrm{~cm}^{3} \\
V^{\prime}=18.566 \mathrm{~cm}^{3}\end{array}$ & $\begin{array}{c}16 \% \mathrm{~S} \\
V_{0}=18.586 \mathrm{~cm}^{3} \\
V^{\prime}=17.581 \mathrm{~cm}^{3}\end{array}$ & $\begin{array}{c}18 \% \mathrm{~s} \\
V_{0}=19.005 \mathrm{~cm}^{3} \\
V^{\prime}=18.074 \mathrm{~cm}^{3}\end{array}$ \\
\hline $\begin{array}{r}\text { atm } \\
10,000 \\
9,000 \\
8,000 \\
7,000 \\
6,000 \\
\\
5,000 \\
4,000 \\
3,000 \\
2,000 \\
1,000\end{array}$ & $\begin{array}{r}0.1037 \\
.0956 \\
.0872 \\
.0779 \\
.0675 \\
.0542 \\
.0395 \\
.0217 \\
.0000 \\
-.0291\end{array}$ & $\begin{array}{r}0.0972 \\
.0899 \\
.0822 \\
.0736 \\
.0641 \\
.0527 \\
.0388 \\
.0214 \\
.0000 \\
-.0272\end{array}$ & $\begin{array}{r}0.0921 \\
.0845 \\
.0764 \\
.0673 \\
.0576 \\
\\
.0471 \\
.0346 \\
.0196 \\
.0000 \\
-.0271\end{array}$ & $\begin{array}{r}0.0910 \\
.0832 \\
.0745 \\
.0652 \\
.0554 \\
\\
.0445 \\
.0323 \\
.0180 \\
.0000 \\
-.0250\end{array}$ \\
\hline & $\begin{array}{c}20 \% \mathrm{~s} \\
V_{0}=18.567 \mathrm{~cm}^{3} \\
V^{\prime}=17.750 \mathrm{~cm}^{3}\end{array}$ & $\begin{array}{c}22 \% \mathrm{~s} \\
V_{0}=18.776 \mathrm{~cm}^{3} \\
V^{\prime}=17.989 \mathrm{~cm}^{3}\end{array}$ & $\begin{array}{c}25 \% \mathrm{~s} \\
V_{0}=18.318 \mathrm{~cm}^{3} \\
V^{\prime}=17.567 \mathrm{~cm}^{3}\end{array}$ & $\begin{array}{c}28 \% \mathrm{~s} \\
V_{0}=18.750 \mathrm{~cm}^{3} \\
V^{\prime}=18.000 \mathrm{~cm}^{3}\end{array}$ \\
\hline $\begin{array}{r}10,000 \\
9,000 \\
8,000 \\
7,000 \\
6,000\end{array}$ & $\begin{array}{r}0.0909 \\
.0831 \\
.0747 \\
.0552 \\
.0551\end{array}$ & $\begin{array}{r}0.0905 \\
.0832 \\
.0747 \\
.0654 \\
.0552\end{array}$ & $\begin{array}{r}0.0929 \\
.0850 \\
.0764 \\
.0668 \\
.0565\end{array}$ & $\begin{array}{r}0.0920 \\
.0844 \\
.0758 \\
.0663 \\
.0562\end{array}$ \\
\hline $\begin{array}{l}5,000 \\
4,000 \\
3,000 \\
2,000 \\
1,000\end{array}$ & $\begin{array}{r}.0438 \\
.0313 \\
.0171 \\
.0000 \\
-.0223\end{array}$ & $\begin{array}{r}.0438 \\
.0313 \\
.0166 \\
.0000 \\
-.0211\end{array}$ & $\begin{array}{r}.0451 \\
.0318 \\
.0168 \\
.0000 \\
-.0203\end{array}$ & $\begin{array}{r}.0447 \\
.0322 \\
.0168 \\
.0000 \\
-.0203\end{array}$ \\
\hline
\end{tabular}

TABLE 6. Compression of natural rubber-sulfur vulcanizates at $81.5^{\circ} \mathrm{C}$

$-\Delta V / V_{0}$

\begin{tabular}{|c|c|c|c|c|}
\hline Pressure & $\begin{array}{c}10 \% \mathrm{~s} \\
V_{0}=19.711 \mathrm{~cm}^{3} \\
V^{\prime}=18.489 \mathrm{~cm}^{3}\end{array}$ & $\begin{array}{c}13 \% \mathrm{~s} \\
V_{0}=20.003 \mathrm{~cm}^{3} \\
V^{\prime}=18.823 \mathrm{~cm}^{3}\end{array}$ & $\begin{array}{c}16 \% \mathrm{~s} \\
V_{0}=18.777 \mathrm{~cm}^{3} \\
V^{\prime}=17.707 \mathrm{~cm}^{3}\end{array}$ & $\begin{array}{c}18 \% \mathrm{~s} \\
V_{0}=19.184 \mathrm{~cm}^{3} \\
V^{\prime}=18.129 \mathrm{~cm}^{3}\end{array}$ \\
\hline $\begin{array}{r}\text { atm } \\
10,000 \\
9,000 \\
8,000 \\
7,000 \\
6,000\end{array}$ & $\begin{array}{r}0.1071 \\
.0999 \\
.0911 \\
.0802 \\
.0695\end{array}$ & $\begin{array}{r}0.1036 \\
.0956 \\
.0875 \\
.0781 \\
.0683\end{array}$ & $\begin{array}{r}0.0975 \\
.0901 \\
.0823 \\
.0729 \\
.0636\end{array}$ & $\begin{array}{r}0.0931 \\
.0857 \\
.0779 \\
.0688 \\
.0592\end{array}$ \\
\hline \multirow[t]{2}{*}{$\begin{array}{l}5,000 \\
4,000 \\
3,000 \\
2,000 \\
1,000\end{array}$} & $\begin{array}{r}.0559 \\
.0409 \\
.0223 \\
.0000 \\
-.0286\end{array}$ & $\begin{array}{r}.0559 \\
.0408 \\
.0228 \\
.0000 \\
-.0283\end{array}$ & $\begin{array}{r}.0527 \\
.0397 \\
.0227 \\
.0000 \\
-.0270\end{array}$ & $\begin{array}{r}.0484 \\
.0354 \\
.0199 \\
.0000 \\
-.0281\end{array}$ \\
\hline & $\begin{array}{c}20 \% \mathrm{~s} \\
V_{0}=18.765 \mathrm{~cm}^{3} \\
V^{\prime}=17.902 \mathrm{~cm}^{3}\end{array}$ & $\begin{array}{c}22 \% \mathrm{~s} \\
V_{0}=19.061 \mathrm{~cm}^{3} \\
V^{\prime}=18.222 \mathrm{~cm}^{3}\end{array}$ & $\begin{array}{c}25 \% \mathrm{~s} \\
V_{0}=18.520 \mathrm{~cm}^{3} \\
V^{\prime}=17.724 \mathrm{~cm}^{3}\end{array}$ & $\begin{array}{c}28 \% \mathrm{~S} \\
V_{0}=18.942 \mathrm{~cm}^{3} \\
V^{\prime}=18.184 \mathrm{~cm}^{3}\end{array}$ \\
\hline $\begin{array}{r}10,000 \\
9,000 \\
8.000 \\
7,000 \\
6,000\end{array}$ & $\begin{array}{r}0.0914 \\
.0843 \\
.0759 \\
.0663 \\
.0578\end{array}$ & $\begin{array}{r}0.0893 \\
.0818 \\
.0736 \\
.0642 \\
.0542\end{array}$ & $\begin{array}{r}0.0905 \\
.0832 \\
.0748 \\
.0649 \\
.0553\end{array}$ & $\begin{array}{r}0.0913 \\
.0838 \\
.0753 \\
.0657 \\
.0558\end{array}$ \\
\hline $\begin{array}{l}5,000 \\
4,000 \\
3,000 \\
2,000 \\
1,000\end{array}$ & $\begin{array}{r}.0461 \\
.0336 \\
.0190 \\
.0000 \\
-.0263\end{array}$ & $\begin{array}{r}.0433 \\
.0305 \\
.0166 \\
.0000 \\
-.0244\end{array}$ & $\begin{array}{r}.0440 \\
.0318 \\
.0174 \\
.0000 \\
-.0214\end{array}$ & $\begin{array}{r}.0442 \\
.0302 \\
.0172 \\
.0000 \\
-.0208\end{array}$ \\
\hline
\end{tabular}


initial volume, $V_{0}$, and the fiducial volume at 2,000 atm, $V^{\prime}$, are given at the head of each column of compressions. Values of $V_{0}$ were measured at $21.0^{\circ}$ $\mathrm{C}$ as described, and the densities so obtained also appear at the head of the columns of data at $21.0^{\circ}$ C. Initial volumes at all other temperatures were calculated, using expansivities obtained from the specific volume-temperature curves for rubbers at 1 atm given by Scott. [3].

The reference volume, $V^{\prime}$, is involved in the calculation of compression and its values are estimated by successive approximations from a generally linear extrapolation of the corresponding calculated compression data. The values of $V^{\prime}$ finally used agree with corresponding values calculated by extrapolation of empirical equations given by Scott [3] to within a few digits in the second significant figure. Attempts to measure the initial compression, $\left(V_{0}-V^{\prime}\right) / V_{0}$ were made, using steel piezometers of the type described by Bridgman [6], and the results corroborated the estimated compression to two significant figures. Higher accuracy was not obtainable with the present experimental setup, in which the rigid requirement of monotonic pressure increase could not be met. The values of initial compression finally used were estimated to only two significant figures but are given in table 7 to three significant figures to correspond to the other data. It is to be noted that small variations even in the second significant figure of the initial compression will have no significant effect on the compressions reported in tables 1 to 6 , inclusive [4, 5].

TABLE 7. Initial compression of rubber-sulfur vulcanizates, 1 to $2,000 \mathrm{~atm}$

$\left(V_{0}-V\right)^{\prime} / V_{0}$

\begin{tabular}{|c|c|c|c|c|}
\hline $\begin{array}{l}\text { Tempera- } \\
\text { ture }\end{array}$ & $10 \% \mathrm{~s}$ & $13 \% \mathrm{~s}$ & $16 \% \mathrm{~s}$ & $18 \% \mathrm{~S}$ \\
\hline $\begin{array}{c}{ }^{\circ} \mathrm{C} \\
10.0 \\
21.0 \\
38.5 \\
50.2 \\
64.0 \\
81.5\end{array}$ & $\begin{array}{r}0.0500 \\
.0510 \\
.0560 \\
.0580 \\
.0590 \\
.0620\end{array}$ & $\begin{array}{r}0.0400 \\
.0420 \\
.0540 \\
.0550 \\
.0570 \\
.0590\end{array}$ & $\begin{array}{r}0.0390 \\
.0390 \\
.0470 \\
.0510 \\
.0540 \\
.0570\end{array}$ & $\begin{array}{r}0.0370 \\
.0370 \\
.0420 \\
.0460 \\
.0490 \\
.0550\end{array}$ \\
\hline & $20 \% \mathrm{~s}$ & $22 \% \mathrm{~s}$ & $25 \% \mathrm{~s}$ & $28 \% \mathrm{~S}$ \\
\hline $\begin{array}{l}10.0 \\
21.0 \\
38.5 \\
50.2 \\
64.0 \\
81.5\end{array}$ & $\begin{array}{r}0.0370 \\
.0420 \\
.0420 \\
.0440 \\
.0460\end{array}$ & $\begin{array}{l}0.0360 \\
.0400 \\
.0410 \\
.0420 \\
.0440\end{array}$ & $\begin{array}{l}0.0360 \\
.0400 \\
.0410 \\
.0410 \\
.0430\end{array}$ & $\begin{array}{l}0.0350 \\
.0400 \\
.0400 \\
.0400 \\
.0420\end{array}$ \\
\hline
\end{tabular}

The values of compression shown in tables 1 to 6 are of the same general order of magnitude as those reported by Adams and Gibson [1] and Bridgman [2]. Two sets of duplicate measurements were made at $21.0^{\circ} \mathrm{C}$ and one at $10^{\circ} \mathrm{C}$. These duplicates indicate that the results are reproducible to within a few digits in the fourth decimal place, as has been shown previously [4]. Because the reduction of temperature to $10^{\circ} \mathrm{C}$ was performed primarily for another purpose, only the rubbers thought to be of particular interest were measured at this temperature. The apparent minimum in compression with increasing sulfur content noted in tables 1 to 6 arises largely from the fact that these data are reckoned from $2,000 \mathrm{~atm}$. If the initial compressions shown in table 7 are added to the corresponding values of tables 1 to 6 , the compression reckoned from $1 \mathrm{~atm}$ is found to decrease with increasing sulfur contents and appears to approach a relatively constant value at high sulfur contents. The effect of sulfur appears to be largely localized in the low-pressure ranges. With increased temperature, compressions also increase, as expected [7].

\section{Empirical Equations}

\subsection{Isotherms}

In the following treatments of the experimental data, the compression is reckoned from $1 \mathrm{~atm}$ rather than from 2,000 atm. Values of compression are obtained by adding corresponding data of table 7 and tables 1 to 6 . Such a procedure introduces into the data the inherent uncertainty contained in the values of table 7 but in no way affects the validity of any equations applied over elevated pressure ranges and in fact can show little over-all error when applied over the complete pressure range studied here.

Ordinary power series of the type

$$
-\Delta V / V_{0}=A+B P+C P^{2}+D P^{3}+
$$

will not adequately represent the experimental data if only a few terms are included in the equation. For harder rubbers such an equation of third degree in $P$ adequately reproduces the data, but for softer rubbers large deviations occur. The addition of terms as high as the fifth power in $P$ does not radically improve the fit for softer rubbers. Accordingly, the equation proposed by Adams and Gibson [1]

$$
-\Delta V / V_{0}=A+B P+C\left(1-e^{-D P}\right)
$$

was used to describe the rubber isotherms of tables 2 to 5 . In this equation the exponential term serves to reproduce the large compressions at low pressures but contributes little at the higher pressures.

The method of fitting such an equation to the data differs from that used by Adams and Gibson [1] and is outlined briefly as follows:

The first difference of eq 2 may be written as

$$
\Delta=B+C D e^{-D P},
$$

which reduces at high pressures to

$$
\Delta \approx B \text {. }
$$

The value of $B$ may, therefore, be estimated by constructing a table of first differences. Using this value of $B$ and taking logarithms of eq (3),

$$
\ln (\Delta-B)=\ln C D-D P \text {. }
$$

A plot of $\ln (\Delta-B)$ as ordinate versus $P$ as abscissa produces a line whose slope is $-D$. This value of $D$ may be substituted into eq (2), and the resulting equations may be fitted to the data by the method of least squares. The coefficients of eq (2) obtained in this manner are tabulated in table 8 for pressure in units of thousands of atmospheres. These coefficients reproduce the experimental data to within 
TABLE 8. Coefficients of isothermal equation ${ }^{1}$ $-\Delta V / V_{0}=A+B P+C\left(1-e^{-D P}\right)$

\begin{tabular}{|c|c|c|c|c|c|c|c|c|}
\hline \multirow{2}{*}{$\begin{array}{l}\text { Temp- } \\
\text { reture }\end{array}$} & \multicolumn{4}{|c|}{$10 \% \mathrm{~S}$} & \multicolumn{4}{|c|}{$13 \% \mathrm{~S}$} \\
\hline & A & B & $\mathrm{C}$ & D & A & B & $\mathrm{C}$ & D \\
\hline \multirow[t]{3}{*}{$\begin{array}{c}\circ C \\
21.0 \\
38.5 \\
50.2 \\
64.0\end{array}$} & $\begin{array}{r}-0.134 \\
-.132 \\
-.135 \\
-.134\end{array}$ & $\begin{array}{r}0.0742 \\
.0705 \\
.0738 \\
.0818\end{array}$ & $\begin{array}{r}0.792 \\
.946 \\
.987 \\
.965\end{array}$ & $\begin{array}{r}0.500 \\
.447 \\
.433 \\
.441\end{array}$ & $\begin{array}{c}-0.156 \\
-.0994 \\
-.128 \\
-.120\end{array}$ & $\begin{array}{r}0.0713 \\
.0706 \\
.0687 \\
.0726\end{array}$ & $\begin{array}{r}0.723 \\
.842 \\
.939 \\
.956\end{array}$ & $\begin{array}{r}0.464 \\
.447 \\
.433 \\
.435\end{array}$ \\
\hline & \multicolumn{4}{|c|}{$16 \% \mathrm{~S}$} & \multicolumn{4}{|c|}{$18 \% \mathrm{~S}$} \\
\hline & A & B & $\mathrm{C}$ & $\mathrm{D}$ & A & B & $\mathrm{C}$ & D \\
\hline \multirow[t]{3}{*}{$\begin{array}{l}21.0 \\
38.5 \\
50.2 \\
64.0\end{array}$} & $\begin{array}{r}-0.0966 \\
-.0377 \\
-.0702 \\
-.110\end{array}$ & $\begin{array}{r}0.0747 \\
.0681 \\
.0660 \\
.0753\end{array}$ & $\begin{array}{r}0.625 \\
.739 \\
.821 \\
.829\end{array}$ & $\begin{array}{r}0.385 \\
.333 \\
.380 \\
.457\end{array}$ & $\begin{array}{r}-0.0419 \\
-.0740 \\
-.0589 \\
-.0550\end{array}$ & $\begin{array}{r}0.0616 \\
.0780 \\
.0700 \\
.0780\end{array}$ & $\begin{array}{r}0.718 \\
.620 \\
.718 \\
.690\end{array}$ & $\begin{array}{r}0.258 \\
.390 \\
.360 \\
.405\end{array}$ \\
\hline & \multicolumn{4}{|c|}{$20 \% \mathrm{~s}$} & \multicolumn{4}{|c|}{$22 \% \mathrm{~S}$} \\
\hline & A & B & $\mathrm{C}$ & $\mathrm{D}$ & A & B & $\mathrm{C}$ & $\mathrm{D}$ \\
\hline $\begin{array}{l}21.0 \\
38.5 \\
50.2 \\
64.0\end{array}$ & $\begin{array}{r}-0.0401 \\
-.0416 \\
-.0583 \\
-.0572\end{array}$ & $\begin{array}{r}0.0652 \\
.0745 \\
.0730 \\
.0749\end{array}$ & $\begin{array}{r}0.686 \\
.641 \\
.674 \\
.682\end{array}$ & $\begin{array}{r}0.263 \\
.333 \\
.335 \\
.350\end{array}$ & $\begin{array}{r}-0.0553 \\
-.0636 \\
-.0444 \\
-.0590\end{array}$ & $\begin{array}{r}0.0653 \\
.0720 \\
.0729 \\
.0731\end{array}$ & $\begin{array}{r}0.690 \\
.673 \\
.666 \\
.685\end{array}$ & $\begin{array}{r}0.268 \\
.324 \\
.310 \\
.330\end{array}$ \\
\hline \multirow{2}{*}{64.0} & \multicolumn{4}{|c|}{$25 \% \mathrm{~S}$} & \multicolumn{4}{|c|}{$28 \% \mathrm{~S}$} \\
\hline & A & B & C & $\mathrm{D}$ & A & B & $\mathrm{C}$ & $\mathrm{D}$ \\
\hline $\begin{array}{l}21.0 \\
38.5 \\
50.2 \\
64.0\end{array}$ & $\begin{array}{r}-0.0880 \\
-.0644 \\
-.0543 \\
-.0622\end{array}$ & $\begin{array}{r}0.0656 \\
.0735 \\
.0724 \\
.0753\end{array}$ & $\begin{array}{r}0.721 \\
.671 \\
.691 \\
.682\end{array}$ & $\begin{array}{r}0.294 \\
.324 \\
.310 \\
.320\end{array}$ & $\begin{array}{r}-0.0816 \\
-.0687 \\
-.0847 \\
-.0766\end{array}$ & $\begin{array}{r}0.0731 \\
.0716 \\
.0745 \\
.0716\end{array}$ & $\begin{array}{r}0.624 \\
.691 \\
.682 \\
.712\end{array}$ & $\begin{array}{r}0.323 \\
.324 \\
.340 \\
.320\end{array}$ \\
\hline
\end{tabular}

1 Coefficients are given for pressure in atm $\times 10^{3}$. a few digits in the fourth decimal place and may be used with confidence between 1 and 10,000 atm. It must be emphasized that the equations cannot be expected to reproduce the exact course of the isotherms between 1 and $1,000 \mathrm{~atm}$ because no experimental data were obtained at pressures below $1,000 \mathrm{~atm}$.

\subsection{Isobars}

The arbitrary nature of the determination of $D$ in eq (2) and the compensation of coefficients that occurs in the least-squares fitting precludes use of the coefficients of table 8 in deriving a general equation of state. To obtain constants that might serve such purposes a different method was used.

From the initial weights, volumes, and compressions given, the specific volumes were calculated at each pressure and temperature measured. To each isobar, so obtained, an equation of the type

$$
V_{s p}=\alpha+\beta t+\gamma t^{2},
$$

where $t$ is the temperature in degrees $\mathrm{C}$, was fitted by the method of least squares. A compilation of the coefficients $\alpha, \beta$, and $\gamma$ so obtained is given in table 9

The coefficients of table 9 reproduce the specific volumes to within $\pm 0.002 \mathrm{~cm}^{3} / \mathrm{g}$ and may be used with confidence in the experimental range. Extrapolation, particularly to higher temperatures, is undesirable because of the nature of the equations and the shape of the isobars. For purely isothermal or isobaric purposes the coefficients of tables 8 and 9 are to be preferred to the following equations of state.

Table 9. Coefficients of isobars ${ }^{1}$

$V_{s p}=\alpha+\beta t+\gamma t^{2}$

\begin{tabular}{|c|c|c|c|c|c|c|}
\hline \multirow[t]{2}{*}{ Pressure } & \multicolumn{3}{|c|}{$\begin{array}{c}10 \% \mathrm{~S} \\
10^{\circ} \mathrm{C}<t<81.5^{\circ} \mathrm{C}\end{array}$} & \multicolumn{3}{|c|}{$\begin{array}{c}13 \% \mathrm{~S} \\
10^{\circ} \mathrm{C}<t<81.5^{\circ} \mathrm{C}\end{array}$} \\
\hline & $\alpha$ & $\beta$ & $\gamma$ & $\alpha$ & $\beta$ & $\gamma$ \\
\hline $\begin{array}{r}10,000 \\
9,000 \\
8,000 \\
7,000 \\
6,000\end{array}$ & $\begin{array}{l}0.8652 \\
.8728 \\
.8807 \\
.8881 \\
.8959\end{array}$ & $\begin{array}{l}-0.7605 \times 10^{-4} \\
-.4684 \\
-.8042 \\
-.5987 \\
-.2012\end{array}$ & $\begin{array}{l}\text { 1. } 178 \times 10^{-6} \\
0.739 \\
1.378 \\
1.772 \\
1.751\end{array}$ & $\begin{array}{r}0.8563 \\
.8635 \\
.8671 \\
.8779 \\
.8874\end{array}$ & $\begin{array}{l}-3.775 \times 10^{-4} \\
-3.577 \\
-2.044 \\
-3.195 \\
-3.718\end{array}$ & $\begin{array}{l}\text { 4. } 022 \times 10^{-6} \\
\text { 3. } 777 \\
\text { 2. } 524 \\
\text { 3. } 799 \\
\text { 4. } 535\end{array}$ \\
\hline \multirow[t]{3}{*}{$\begin{array}{r}5,000 \\
4,000 \\
3,000 \\
2,000 \\
1,000 \\
1\end{array}$} & $\begin{array}{l}.9062 \\
.9158 \\
.9265 \\
.9415 \\
.9662 \\
.9904\end{array}$ & $\begin{array}{l}-.1381 \\
+1.276 \\
+3.391 \\
+5.206 \\
+6.699 \\
+7.389\end{array}$ & $\begin{array}{r}2.304 \\
1.514 \\
0.190 \\
-.606 \\
-1.777 \\
-1.135\end{array}$ & $\begin{array}{l}.8971 \\
.9082 \\
.9199 \\
.9335 \\
.9525 \\
.9652\end{array}$ & $\begin{array}{l}-3.323 \\
-3.469 \\
-2.704 \\
-9.530 \\
+1.750 \\
+5.502\end{array}$ & $\begin{array}{l}\text { 4. } 418 \\
\text { 5. } 302 \\
\text { 5. } 379 \\
\text { 4. } 602 \\
\text { 2. } 786 \\
0.910\end{array}$ \\
\hline & \multicolumn{3}{|c|}{$\begin{array}{c}16 \% \mathrm{~S} \\
10^{\circ} \mathrm{C}<t<81.5^{\circ} \mathrm{C}\end{array}$} & \multicolumn{3}{|c|}{$\begin{array}{c}18 \% \mathrm{~S} \\
21^{\circ} \mathrm{C}<t<81.5^{\circ} \mathrm{C}\end{array}$} \\
\hline & $\alpha$ & $\beta$ & $\gamma$ & $\alpha$ & $\beta$ & $\gamma$ \\
\hline $\begin{array}{r}10,000 \\
9,000 \\
8,000 \\
7,000 \\
6,000\end{array}$ & $\begin{array}{r}0.8259 \\
.8328 \\
.8392 \\
.8447 \\
.8540\end{array}$ & $\begin{array}{l}\text { 1. } 742 \times 10^{-4} \\
\text { 1. } 827 \\
\text { 2. } 291 \\
\text { 3. } 699 \\
\text { 3. } 406\end{array}$ & $\begin{array}{l}-1.218 \times 10^{-6} \\
-1.310 \\
-1.644 \\
-2.883 \\
-2.443\end{array}$ & $\begin{array}{r}0.8185 \\
.8297 \\
.8325 \\
.8322 \\
.8497\end{array}$ & $\begin{array}{l}-1.395 \times 10^{-4} \\
-2.749 \\
-0.797 \\
+3.010 \\
-0.900\end{array}$ & $\begin{array}{l}\text { 2. } 696 \times 10^{-6} \\
3.794 \\
1.894 \\
-1.424 \\
2.411\end{array}$ \\
\hline $\begin{array}{r}5,000 \\
4,000 \\
3,000 \\
2,000 \\
1,000 \\
1\end{array}$ & $\begin{array}{l}.8635 \\
.8761 \\
.8883 \\
.9030 \\
.9175 \\
.9354\end{array}$ & $\begin{array}{l}\text { 3. } 815 \\
3.298 \\
3.096 \\
\text { 3. } 110 \\
6.119 \\
6.721\end{array}$ & $\begin{array}{l}-2.924 \\
-2.146 \\
-1.238 \\
-0.104 \\
-2.035 \\
-0.954\end{array}$ & $\begin{array}{l}.8610 \\
.8719 \\
.8865 \\
.9018 \\
.9141 \\
.9298\end{array}$ & $\begin{array}{l}-1.055 \\
-0.992 \\
-2.009 \\
-2.275 \\
+1.530 \\
+4.716\end{array}$ & $\begin{array}{l}2.421 \\
2.494 \\
3.945 \\
4.820 \\
3.923 \\
5.204\end{array}$ \\
\hline
\end{tabular}


Table 9. Coefficients of isobars ${ }^{1}$-Continued

\begin{tabular}{|c|c|c|c|c|c|c|}
\hline \multicolumn{7}{|c|}{$V_{s p}=\alpha+\beta t+\gamma t^{2}$} \\
\hline & \multicolumn{3}{|c|}{$\begin{array}{c}20 \% \mathrm{~S} \\
10^{\circ} \mathrm{C}<t<81.5^{\circ} \mathrm{C}\end{array}$} & \multicolumn{3}{|c|}{$\begin{array}{c}22 \% \mathrm{~S} \\
21^{\circ} \mathrm{C}<t<81.5^{\circ} \mathrm{C}\end{array}$} \\
\hline & $\alpha$ & $\beta$ & $\gamma$ & $\alpha$ & $\beta$ & $\gamma$ \\
\hline $\begin{array}{r}10,000 \\
9,000 \\
8,000 \\
7,000 \\
6,000\end{array}$ & $\begin{array}{l}0.8071 \\
.8137 \\
.8201 \\
.8269 \\
.8344\end{array}$ & $\begin{array}{l}-2.077 \times 10^{-4} \\
-1.754 \\
-1.226 \\
-0.802 \\
-.056\end{array}$ & $\begin{array}{l}\text { 4. } 076 \times 10^{-6} \\
\text { 3. } 736 \\
\text { 3. } 332 \\
\text { 3. } 146 \\
\text { 2. } 312\end{array}$ & $\begin{array}{l}0.8082 \\
.8130 \\
.8212 \\
.8323 \\
.8390\end{array}$ & $\begin{array}{l}-7.061 \times 10^{-4} \\
-6.310 \\
-6.572 \\
-7.845 \\
-7.059\end{array}$ & $\begin{array}{l}\text { 8. } 899 \times 10^{-6} \\
\text { 8. } 303 \\
\text { 8. } 615 \\
\text { 9. } 856 \\
9.227\end{array}$ \\
\hline $\begin{array}{r}5,000 \\
4,000 \\
3,000 \\
2,000 \\
1,000 \\
1\end{array}$ & $\begin{array}{l}.8442 \\
.8563 \\
.8683 \\
.8824 \\
.9048 \\
.9142\end{array}$ & $\begin{array}{r}+.117 \\
+.239 \\
+.260 \\
+.089 \\
-4.457 \\
+1.851\end{array}$ & $\begin{array}{l}\text { 2. } 320 \\
2.270 \\
\text { 2. } 380 \\
\text { 3. } 158 \\
9.790 \\
2.687\end{array}$ & $\begin{array}{l}.8469 \\
.8587 \\
.8699 \\
.8841 \\
.9004 \\
.9128\end{array}$ & $\begin{array}{l}-5.809 \\
-6.255 \\
-5.308 \\
-5.249 \\
-5.020 \\
-2.983\end{array}$ & $\begin{array}{l}8.008 \\
8.580 \\
7.712 \\
7.874 \\
8.452 \\
6.932\end{array}$ \\
\hline \multirow{2}{*}{$\begin{array}{r}1,000 \\
1\end{array}$} & \multicolumn{3}{|c|}{$\begin{array}{c}25 \% \mathrm{~S} \\
21^{\circ} \mathrm{C}<t<81.5^{\circ} \mathrm{C}\end{array}$} & \multicolumn{3}{|c|}{$\begin{array}{c}28 \% \mathrm{~S} \\
21^{\circ} \mathrm{C}<t<81.5^{\circ} \mathrm{C}\end{array}$} \\
\hline & $\alpha$ & $\beta$ & $\gamma$ & $\alpha$ & $\beta$ & $\gamma$ \\
\hline $\begin{array}{r}10.000 \\
9,000 \\
8,000 \\
7,000 \\
6,000\end{array}$ & $\begin{array}{r}0.7806 \\
.7946 \\
.7981 \\
.8061 \\
.8143\end{array}$ & $\begin{array}{l}-2.048 \times 10^{-4} \\
-4.770 \\
-3.171 \\
-3.234 \\
-2.952\end{array}$ & $\begin{array}{l}\text { 3. } 114 \times 10^{-6} \\
5.450 \\
3.968 \\
\text { 4. } 225 \\
\text { 4. } 004\end{array}$ & $\begin{array}{r}0.7690 \\
.7760 \\
.7831 \\
.7953 \\
.7990\end{array}$ & $\begin{array}{l}-2.407 \times 10^{-4} \\
-2.407 \\
-2.527 \\
-4.393 \\
-1.925\end{array}$ & $\begin{array}{l}\text { 3. } 318 \times 10^{-6} \\
\text { 3. } 318 \\
\text { 3. } 629 \\
\text { 5. } 384 \\
\text { 3. } 041\end{array}$ \\
\hline $\begin{array}{r}5,000 \\
4,000 \\
3,000 \\
2,000 \\
1,000 \\
1\end{array}$ & $\begin{array}{l}.8232 \\
.8334 \\
.8458 \\
.8614 \\
.8813 \\
.8901\end{array}$ & $\begin{array}{l}-2.252 \\
-1.851 \\
-1.567 \\
-1.851 \\
-2.996 \\
+0.217\end{array}$ & $\begin{array}{l}\text { 3. } 325 \\
2.992 \\
2.659 \\
2.992 \\
4.399 \\
2.030\end{array}$ & $\begin{array}{l}.8068 \\
.8189 \\
.8268 \\
.8434 \\
.8589 \\
.8709\end{array}$ & $\begin{array}{r}-1.174 \\
-2.118 \\
+5.117 \\
-0.451 \\
-.146 \\
+1.698\end{array}$ & $\begin{array}{l}2.445 \\
3.689 \\
0.861 \\
2.073 \\
2.049 \\
0.798\end{array}$ \\
\hline
\end{tabular}

1 Temperature in degrees $\mathrm{C}$ in the specified range.

\subsection{Equations of State}

To obtain a PVT equation of state the coefficients $\beta$ and $\gamma$ given in table 9 for a particular rubber were each fitted to an equation of the type

$$
\beta \text { or } \gamma=a+b P+c P^{2}+d P^{3} .
$$

The values of $a, b, c$, and $d$ so obtained were used to recalculate values of $\beta$ and $\gamma$ at each pressure. The calculated $\beta$ and $\gamma$ values were used with the observed $V_{s p}$ values to calculate corrected values of $\alpha$, which were then fitted by least squares to a cubic in pressure. The correction applied to values of $\alpha$ was in most instances negligible. However, the values of $\alpha$ are not designed to correspond to the values of table 9 but rather to produce correct values of $V_{s p}$. As $\alpha$, $\beta$, and $\gamma$ have been defined as coefficients of $t$ in eq 6 , the 12 values of $a, b, c$, and $d$ may be utilized to formulate an empirical equation of state. These equations and their known limits of accuracy follow:

For 10 percent of sulfur:

$$
\begin{aligned}
V_{s p}= & 0.9904-3.107 \times 10^{-5} P+3.688 \times 10^{-9} P^{2}- \\
& 1.857 \times 10^{-13} P^{3}+\left(7.389 \times 10^{-4}-9.098 \times 10^{-9} P-\right. \\
& \left.1.926 \times 10^{-12} P^{2}+2.110 \times 10^{-16} P^{3}\right) t+(-1.135 \times \\
& 10^{-6}+7.591 \times 10^{-10} P-2.045 \times 10^{-14} P^{2}+ \\
& \left.3.764 \times 10^{-18} P^{3}\right) t^{2}
\end{aligned}
$$

$1<P<10,000$ atm; $10^{\circ} \mathrm{C}>t<81.5^{\circ} \mathrm{C}$; deviatio ${ }^{\mathrm{n}}$ from observed values increases at highest temperature and lowest pressure. Except at highest temperature and lowest pressure, deviation is \pm 0.45 percent.

For 13 percent of sulfur:

$$
\begin{aligned}
V_{s p}= & 0.9652-1.648 \times 10^{-5} P+5.786 \times 10^{-10} P^{2}- \\
& 1.551 \times 10^{-15} P^{3}+\left(5.502 \times 10^{-4}-4.654 \times\right. \\
& \left.10^{-7} P+7.677 \times 10^{-11} P^{2}-3.958 \times 10^{-15} P^{3}\right) t+ \\
& \left(0.9103 \times 10^{-6}+2.899 \times 10^{-9} P-5.939 \times\right. \\
& \left.10^{-13} P^{2}+3.355 \times 10^{-17} P^{3}\right) t^{2}
\end{aligned}
$$

$1<\mathrm{P}<10,000 \mathrm{~atm} ; 10^{\circ} \mathrm{C}<t<81.5^{\circ} \mathrm{C}$; maximum deviation from observed values \pm 0.33 percent.

For 16 percent of sulfur:

$$
\begin{aligned}
V_{s p}= & 0.9354-1.694 \times 10^{-5} P+4.338 \times 10^{-10} P^{2}+ \\
& 1.840 \times 10^{-14} P^{3}+\left(6.72 \times 10^{-4}-1.953 \times 10^{-7} P+\right. \\
& \left.3.617 \times 10^{-11} P^{2}-2.207 \times 10^{-15} P^{3}\right) t+(-0.954 \times \\
& 10^{-6}-4.336 \times 10^{-10} P+4.623 \times 10^{-14} P^{2}- \\
& \left.5.74 \times 10^{-19} P^{3}\right) t^{2} .
\end{aligned}
$$


$1<P<10,000$ atm; $10^{\circ} \mathrm{C}<t<81.5^{\circ} \mathrm{C}$; maximum deviation from observed values \pm 0.37 percent.

For 18 percent of sulfur:

$V_{s p}=0.9298-1.633 \times 10^{-5} \mathrm{P}+3.15 \times 10^{-10} P^{2}+2.29 \times$

$$
10^{-14} P^{3}+\left(4.716 \times 10^{-4}-4.604 \times 10^{-7} P+\right.
$$$$
\left.9.934 \times 10^{-11} P^{2}-6.078 \times 10^{-15} P^{3}\right) t+(5.204 \times
$$$$
10^{-6}-9.780 \times 10^{-11} P-2.082 \times 10^{-13} P^{2}+
$$$$
\left.2.011 \times 10^{-17} P^{3}\right) t^{2} .
$$

$1<P<10,000$ atm; $21^{\circ} \mathrm{C}<t<81.5^{\circ} \mathrm{C}$; equation deviates sharply at highest temperature at lowest pressure. Except at highest temperature and lowest pressure, deviation from observed values is \pm 0.75 percent.

For 20 percent of sulfur:

$V_{s D}=0.9142-2.084 \times 10^{-5} P+1.554 \times 10^{-9} P^{2}-$

$$
4.78 \times 10^{-14} P^{3}+\left(1.851 \times 10^{-4}-7.855 \times 10^{-8} P+\right.
$$$$
\left.1.859 \times 10^{-11} P^{2}-1.559 \times 10^{-15} P^{3}\right) t+(2.687 \times
$$$$
10^{-6}+1.182 \times 10^{-9} P-4.099 \times 10^{-13} P^{2}+2.967 \times
$$$$
\left.10^{-17} P^{3}\right) t^{2}
$$

$1<P<10,000 \mathrm{~atm} ; 10^{\circ} \mathrm{C}<t<81.5^{\circ} \mathrm{C}$; maximum deviation from observed values, \pm 0.87 percent.

For 22 percent of sulfur:

$V_{s p}=0.9128-1.483 \times 10^{-5} \mathrm{P}+2.90 \times 10^{-10} P^{2}+1.45 \times$

$10^{-14} P^{3}+\left(-2.98 \times 10^{-4}-1.336 \times 10^{-7} P+\right.$

$\left.1.543 \times 10^{-11} P^{2}-5.85 \times 10^{-16} P^{3}\right) t+(6.932 \times$

$10^{-6}+3.574 \times 10^{-10} \mathrm{P}+1.575 \times 10^{-14} \mathrm{P}^{2}-3.506 \times$

$$
\left.10^{-18} P^{3}\right) t^{2}
$$

$1<P<10,000$ atm; $21^{\circ} \quad \mathrm{C}<t<81.5^{\circ} \mathrm{C}$; maximum deviation from observed values, \pm 0.37 percent.

For 25 percent of sulfur:

$V_{s y}=0.8901-1.562 \times 10^{-5} \mathrm{P}+5.11 \times 10^{-10} \mathrm{P}^{2}+1.1 \times$

$10^{-15} P^{3}+\left(0.217 \times 10^{-4}-1.054 \times 10^{-7} P+\right.$

$\left.1.064 \times 10^{-11} P^{2}-3.436 \times 10^{-16} P^{3}\right) t+(2.030 \times$

$10^{-6}+4.047 \times 10^{-10} \mathrm{P}-2.59 \times 10^{-15} \mathrm{P}^{2}-1.86 \times$

$\left.10^{-18} P^{3}\right) t^{2}$
$1<P<10,000$ atm; $21^{\circ} \quad \mathrm{C}<t<81.5^{\circ} \quad \mathrm{C}$; maximum deviation from observed values \pm 0.25 percent. Most figures show deviations of \pm 0.12 percent or less.

For 28 percent of sulfur:

$V_{s p}=0.8709-1.486 \times 10^{-5} \mathrm{P}+5.61 \times 10^{-10} \mathrm{P}^{2}-$

$8.8 \times 10^{-15} P^{3}+\left(1.698 \times 10^{-4}-1.264 \times 10^{-7} P+\right.$

$\left.1.017 \times 10^{-11} P^{2}-1.659 \times 10^{-16} P^{3}\right) t+(0.798 \times$

$10^{-6}+7.786 \times 10^{-10} \mathrm{P}-4.074 \times 10^{-14} \mathrm{P}^{2}$

$\left.1.217 \times 10^{-18} P^{3}\right) t^{2}$

$1<P<10,000$ atm; $21^{\circ} \quad \mathrm{C}<t<81.5^{\circ} \quad \mathrm{C}$; maximum deviation from observed values, \pm 0.37 percent.

The derivation of eq (8) to (15) represents the main objective of the present report. The equations have not been examined critically in a mathematical sense. Physically, the signs and courses of the first partial derivatives are in agreement with expectations, and no singularities are observed in the experimental range. It is believed that these equations may be used with confidence for subsequent calculation purposes where either the isobaric or isothermal equations cannot be used.

It appears that the process of generalization may be carried one step further whereby each of the 12 coefficients in eq (8) to (15) can be related to a linear or quadratic expression of sulfur content. This would reduce the 8 equations containing 96 coefficients to a single general equation of at most 36 coefficients. Such a reduction may be useful for some purposes but can be deferred until such time as data are available on rubbers of lower sulfur content.

A valid comparison of the present data and those of Scott is not feasible as the experimental pressure ranges do not overlap. Generalizing on the basis of a limited number of tests that have been made, the following observations may be made: Extrapolation of Scott's equations to $1,000 \mathrm{~atm}$ produces data that are in generally good agreement with the data observed here. Such an extrapolation, it is to be noted, represents a rather considerable extension of the pressure interval employed by Scott.

For information concerning the behavior of rubber at low pressures, the data of Scott are considered preferable to those reported here, which may not be expected to closely reproduce the PVT behavior below 1,000 atm. For pressures above 1,000 atm, the equations given by Scott cannot be considered reliable, and the present equations are to be preferred.

The author is indebted to W. H. Leser for valuable suggestions concerning the fitting of empirical equations to the data. 


\section{References}

[1] L. H. Adams and R. E. Gibson, J. Wash. Acad. Sci. 20, 213 (1930)

[2] P. W. Bridgman, Proc. Am. Acad. Arts Sci. \%6, 9 (1945).

[3] A. H. Scott, J. Research NBS 14, 100 (1935) RP760.

[4] C. E. Weir, J. Research NBS 46, 207 (1951) RP2192.
[5] C. E. Weir, J. Research NBS 45, 468 (1950) RP2160.

[6] P. W. Bridgman, Proc. Am. Acad. Arts Sci. 47, 347 (1911).

[7] P. W. Bridgman, The physics of high pressure (G. Bell \& Sons, Ltd., London, 1949).

Washington, November 6, 1952. 